\title{
A COMPARISON OF MEAN CONCENTRATIONS IN A DIFFUSION PROBLEM
}

\author{
by A. BROWN \\ (Received 19th October 1979)
}

\section{Introduction}

In some biological problems, a parasite-host system is immersed in a toxic solution in order to kill off the parasite while leaving the host as little affected as possible. A problem of this type was considered by Clements and Edelstein (2), who treated both the host and the parasite as cylindrical in shape. In a separate paper Clements (1) considered the corresponding problem where the parasite is spherical and the host cylindrical. In both cases, the concentration of the toxic solution at the boundary is taken as having a constant value, $c$, and the penetration of the poison into the host and parasite is treated as a linear diffusion problem with an appropriate diffusion coefficient. It is assumed also that the host and the parasite are free of the toxic substance initially. The process is terminated when the average concentration in the parasite reaches a lethal level, $\tau$, and the problem is to see how $M$, the average concentration in the host, is affected by the choice of $c$ (for a given value of $\tau$ ).

The effect of the size of the organisms and of their diffusion coefficients can be concentrated into a single parameter, $\rho$, and Clements and Edelstein obtained a number of results for the way in which $M$ varies with $c$, for different values of $\rho$. For the cylinder-sphere comparison, Clements obtained some simular results and made a general conjecture about the way in which $M$ varies. The present paper extends these results, particularly in the case where $c$ is large compared with $\tau$, and shows that Clements' conjecture has to be modified.

Section 2 gives the basic equations for diffusion into a cylindrical organism and for the cylinder-cylinder comparison. It is convenient to write $M$ in terms of $\rho$ and a time-like variable, $t$, with $t \rightarrow 0$ as $c / \tau \rightarrow \infty$. A Laplace transform approach is used to obtain $M$ as a power series in $t^{1 / 2}$, for $t$ small (Section 3) and the way in which $M$ varies with $\rho$ and $t$ is discussed in detail in Section 4. In Section 5, similar results to those in Sections 2 and 3 are obtained for diffusion into a spherical organism and in Sections 6 to 8 detailed results are developed for the cylinder-sphere comparison, i.e. where the host organism is cylindrical and the parasite spherical. In Section 9, a brief statement is made of the results for the case where the host organism is spherical and the parasite is cylindrical.

\section{Diffusion into cylindrical organism}

For an infinitely long cylinder of radius $a$, the concentration of the toxic material, $C(r, t)$ at distance $r$ from the axis of the cylinder and for time $t>0$ is taken as satisfying 
the diffusion equation

$$
\frac{\partial C}{\partial t}=k\left(\frac{\partial^{2} C}{\partial r^{2}}+\frac{1}{r} \frac{\partial C}{\partial r}\right)
$$

and the boundary conditions

$$
C(r, 0)=0, \quad C(a, t)=c .
$$

The diffusion coefficient, $k$, is taken as constant. Solving by separation of variables and averaging over $r$ gives the average concentration as

$$
\bar{C}(t)=c G_{1}\left(t_{1}\right),
$$

where

$$
t_{1}=k t / a^{2}
$$

and

$$
G_{1}\left(t_{1}\right)=1-4 \sum_{p=1}^{\infty}\left(1 / \lambda_{p}\right)^{2} \exp \left(-\lambda_{p}^{2} t_{1}\right) .
$$

The constants $\lambda_{\mathrm{p}}$ are the positive zeros of the Bessel function $J_{0}(x)$, with $0<\lambda_{1}<\lambda_{2}<$ $\lambda_{3}<\ldots$. The function $G_{1}\left(t_{1}\right)$ can be thought of as a standardised solution of the problem, valid for the special case where $a=1, k=1$, and $c=1$. The ratio $t_{1} / t=k / a^{2}$ is the time scale factor required to relate the given problem to the standardised solution. (The notation here is not exactly the same as in the paper by Clements and Edelstein but it is useful later to have $G_{1}\left(t_{1}\right)$ in this standardised form.) From the physics of the problem, $G_{1}$ must be a monotonic increasing function of $t_{1}$, with $G_{1}(0)=0$ and $G_{1} \rightarrow 1$ as $t_{1} \rightarrow \infty$. If we write $G_{1}^{\prime}\left(t_{1}\right)=H_{1}\left(t_{1}\right)$, then $H_{1}\left(t_{1}\right)$ is the average rate at which the toxic substance is crossing the boundary into the organism (in the standardised problem), since it is this diffusion across the boundary which produces the increase in $G_{1}$. As the diffusion rate is proportional to the radial gradient of the concentration, we can expect $H_{1}$ to be unbounded initially because of the discontinuity in the concentration at the boundary of the cylinder. From equation (2.5).

$$
H_{1}\left(t_{1}\right)=4 \sum_{p=1}^{\infty} \exp \left(-\lambda_{p}^{2} t_{1}\right)
$$

for $t_{1} \geqq t_{0}>0$. Clearly, $H_{1}$ is monotonic decreasing, with $H_{1} \rightarrow 0$ as $t_{1} \rightarrow \infty$.

For a cylinder of radius $a_{1}$ and diffusion coefficient $k_{1}$, we can use the same standardised solution and write

$$
\bar{C}_{1}(t)=\text { average concentration at time } t=c G_{1}\left(t_{2}\right)
$$

where

$$
t_{2}=k_{1} t /\left(a_{1}\right)^{2}
$$

The ratio

$$
\rho=t_{1} / t_{2}=\left(k / k_{1}\right)\left(a_{1} / a\right)^{2}
$$

relates the time scales appropriate to each organism. If $\bar{C}_{1}=\tau$ when $t_{2}=T_{2}$, then the corresponding value for $\bar{C}$ is $c G_{1}\left(\rho T_{2}\right)$, since $t_{1}=\rho t_{2}$. Thus if we take $\bar{C}_{1}$ as the average 
concentration of the toxic in the parasite and $\bar{C}$ as the average concentration in the host,

$$
\begin{aligned}
M & =\text { value of } \bar{C} \text { when } \bar{C}_{1} \text { reaches value } \tau \\
& =c G_{1}\left(\rho T_{2}\right)=\tau G_{1}\left(\rho T_{2}\right) / G_{1}\left(T_{2}\right),
\end{aligned}
$$

since

$$
\tau=\bar{C}_{1}\left(T_{2}\right)=c G_{1}\left(T_{2}\right) \text {. }
$$

The value of $T_{2}$ depends on the choice of $c$, in the sense that the larger $c$ becomes the less time is required to raise $\bar{C}_{1}$ to the level $\tau$. As $c \rightarrow \infty, T_{2}$ must tend to zero and as $c \rightarrow \tau$ from above, $T_{2}$ will tend to infinity. Clements and Edelstein treat $M$ as a function of $c$, with $\rho$ as a parameter, but for the detailed discussion below it was found easier to work with $M$ in terms of $T_{2}$. Since $d T_{2} / d c$ is negative, it is easy to change results concerning the sign of $d M / d T_{2}$ to results in terms of the sign of $d M / d c$.

To simplify the notation, we can replace $T_{2}$ by $t$ in equation (2.10) and write

$$
M(t)=\tau G_{1}(\rho t) / G_{1}(t) .
$$

With this notation, the main results obtained by Clements and Edelstein are

(i) $M \rightarrow \tau \sqrt{ } \rho$ as $t \rightarrow 0$,

(ii) $M^{\prime}>0$ for $\rho<1, t>\left(1 / \lambda_{1}\right)^{2}$,

(iii) $M^{\prime}<0$ for $\rho>1, t>\left(1 / \lambda_{1}\right)^{2}$.

They indicate that the restriction on $t$ in (ii) and (iii) is probably not required and this is confirmed in Sections 3 and 4 below.

\section{Approximations to $H_{1}, G_{1}$ and $M$ for $t$ small}

Equations (2.5) and (2.6) are inconvenient to use when $t_{1}$ is small. To obtain an alternative form, let

$$
h_{1}(s)=\int_{0}^{\infty} H_{1}(t)\{\exp (-s t)\} d t=\text { Laplace transform of } H_{1} .
$$

Integrating term by term,

$$
h_{1}(s)=4 \sum_{p=1}^{\infty}\left(s+\lambda_{p}^{2}\right)^{-1},
$$

a series which converges for $\operatorname{Re}(s)>0$, since

$$
\lambda_{\mathrm{p}}=\{p-(1 / 4)+\varepsilon(p)\} \pi,
$$

where the "correction terms" $\varepsilon(p)$ form a monotonic decreasing sequence of positive quantities, with $\varepsilon(1)<0.0155$. Watson (5) gives numerical values for $\lambda_{1}$ to $\lambda_{40}$ in Table VII at the end of his book and he discusses the form of $\varepsilon(p)$ in more detail in $\$ 15.52$. In $\$ 15.41$, he obtains an infinite series for $J_{v+1}(z) / J_{v}(z)$, using Mittag-Leffler's theorem 
(4, \$5.10), and the same technique can be used to deduce that for $\operatorname{Re}(z)>0$

$$
\sum_{p=1}^{\infty} \frac{2 z}{z^{2}+\lambda_{p}^{2}}=\frac{I_{1}(z)}{I_{0}(z)}
$$

where $I_{n}(z)$ is the modified Bessel function of order $n$. (The zeros of $I_{0}(z)$ lie on the imaginary axis at $\pm i \lambda_{p}$ and the essential restriction on $z$ in equation (3.4) is that $z$ does not coincide with one of these zeros.) If we think of $s$ as real and positive in equation (3.2), we can make use of equation (3.4) to write

$$
h_{1}(s)=\{2 / \sqrt{ } s\} I_{1}(\sqrt{ } s) / I_{0}(\sqrt{ } s\},
$$

an expression which can be expanded in powers of $s^{-1 / 2}$ for $s$ large. This can be done by using asymptotic expansions for $I_{1}(x)$ and $I_{0}(x)$, as given by Erdelyi et al. $(3$, p. 86) or by Watson $(5, \S 7.23)$. An alternative method is to start from the equation $x y^{\prime \prime}+y^{\prime}-x y=0$, which has $y=I_{0}(x)$ as a solution, and to substitute $w=y^{\prime} / y$. The equation for $w$ can be written as

$$
w^{\prime}=1-w^{2}-(w / x)
$$

and it has an asymptotic solution, for $x \rightarrow \infty$, of the form

$$
w=1-\sum_{r=1}^{\infty}\left(a_{r} / x^{r}\right),
$$

which turns out to be exactly what we want for $I_{1}(x) / I_{0}(x)$. (Note that $I_{0}^{\prime}=I_{1}$.) In practice, it is easier to evaluate $a_{1}, a_{2}, a_{3}, \ldots$ in turn from equations (3.6) and (3.7) than to write down the expansions for $I_{1}(x)$ and $I_{0}(x)$ separately and then use the binomial theorem to get an expansion for $I_{1}(x) / I_{0}(x)$. This gives

$$
\begin{aligned}
& a_{1}=1 / 2, \quad a_{2}=a_{3}=1 / 8, \quad a_{4}=25 / 128, \quad a_{5}=13 / 32, \\
& a_{6}=1073 / 1024, \quad a_{7}=103 / 32, \quad a_{8}=375733 / 32768 .
\end{aligned}
$$

Thus for $s$ large,

$$
h_{1}(s)=(2 / \sqrt{ } s)\left\{1-\sum_{r=1}^{7} a_{r} s^{-r / 2}\right\}+O\left(s^{-9 / 2}\right)
$$

and correspondingly we can expect the behaviour of $H_{1}(t)$ for small $t$ to be given by

$$
H_{1}^{*}(t)=\{2 / \sqrt{ }(\pi t)\}-\sum_{r=1}^{7} b_{r} t^{(r-1) / 2}+O\left(t^{7 / 2}\right)
$$

where

$$
b_{r}=2 a_{r} / \Gamma\{(r+1) / 2\}
$$

Integrating this expression from zero to $t$ gives a corresponding approximation to $G_{1}(t)$ for $t$ small, i.e.

$$
G_{1}^{*}(t)=4\{\sqrt{ }(t / \pi)\}-\sum_{r=1}^{7} c_{r} t^{(r+1) / 2}+O\left(t^{9 / 2}\right)
$$


Table 1. Comparison of $G_{1}(t), H_{1}(t)$ and $K(t)$ with power series approximations for $t$ small.

\begin{tabular}{ccccccc}
\hline$t$ & $G_{1}(t)$ & $G_{1}^{*}(t)$ & $H_{1}(t)$ & $H_{1}^{*}(t)$ & $K(t)$ & $K^{*}(t)$ \\
\hline 0.02 & 0.2986 & 0.2986 & 6.9329 & 6.9329 & 0.4644 & 0.4644 \\
0.04 & 0.4096 & 0.4096 & 4.5722 & 4.5722 & 0.4465 & 0.4465 \\
0.06 & 0.4894 & 0.4894 & 3.5157 & 3.5159 & 0.4310 & 0.4310 \\
0.08 & 0.5529 & 0.5529 & 2.8780 & 2.8787 & 0.4164 & 0.4165 \\
0.10 & 0.6058 & 0.6059 & 2.4356 & 2.4376 & 0.4020 & 0.4023 \\
\hline
\end{tabular}

where

$$
c_{r}=2 b_{r} /(r+1)=2 a_{r} / \Gamma\{(r+3) / 2\}
$$

A numerical check gave the values in Table 1 for $G_{1}(t), G_{1}^{*}(t), H_{1}(t), H_{1}^{*}(t)$ and for two related functions $K(t)$ and $K^{*}(t)$ which are required in Section 4. (In fact, $K(t)=t H_{1}(t) / G_{1}(t)$ and $K^{*}(t)$ is the corresponding approximation $t H_{1}^{*}(t) / G_{1}^{*}(t)$.) It will be seen that the power series approximations give 4-figure accuracy up to about $t=0.08$ and are beginning to diverge at $t=0.1$.

If we write $G_{1}^{*}(t)$ in the form

$$
G_{1}^{*}(t)=4\{\sqrt{ }(t / \pi)\}\left(1-d_{1} u-d_{2} u^{2}-d_{3} u^{3}-\ldots\right),
$$

where $u=\sqrt{ } t$ and the coefficients $d_{r}$ are all positive, then an expansion in series as far as $u^{4}$ terms gives

$$
\begin{aligned}
M_{1}^{*}= & \text { approximation to } M(t) \text { for } t \text { small } \\
= & \tau G_{1}^{*}(\rho t) / G_{1}^{*}(t)=\tau G_{1}^{*}\left(\sigma^{2} u^{2}\right) / G_{1}^{*}\left(u^{2}\right) \\
= & \sigma \tau\left[1+d_{1} u(1-\sigma)+\left\{d_{2}\left(1-\sigma^{2}\right)+d_{1}^{2}(1-\sigma)\right\} u^{2}\right. \\
& +\left\{d_{3}\left(1-\sigma^{3}\right)+d_{1} d_{2}\left(1-\sigma^{2}\right)+d_{1} d_{2}(1-\sigma)+d_{1}^{3}(1-\sigma)\right\} u^{3} \\
& +\left\{d_{4}\left(1-\sigma^{4}\right)+d_{1} d_{3}\left(1-\sigma^{3}\right)+\left(d_{2}^{2}+d_{1}^{2} d_{2}\right)\left(1-\sigma^{2}\right)\right. \\
& \left.\left.+\left(2 d_{1}^{2} d_{2}+d_{1} d_{3}+d_{1}^{4}\right)(1-\sigma)\right\} u^{4}\right],
\end{aligned}
$$

where $\sigma=\sqrt{ } \rho$. From equation (3.15), $M_{1}^{*} \rightarrow \tau \sqrt{ } \rho$ as $t \rightarrow 0$, in agreement with equation (2.13), and also $M_{1}^{*}$ approaches the limit from above for $0<\rho<1$ and from below for $\rho>1$.

The result above is an example of a more general property which will be useful later. If we have

$$
F(t)=\{1-A(t)\} /\{1-B(t)\}
$$

with

$$
\begin{aligned}
& A^{\prime}(t)>B^{\prime}(t)>0 \text { for } 0 \leqq t \leqq t_{0} \text {, } \\
& A(0)=0, \quad B(0)=0, \quad A\left(t_{0}\right)<1,
\end{aligned}
$$

then $F(t)$ is monotonic decreasing for $0 \leqq t \leqq t_{0}$. One way of seeing that this statement is true is to re-write equation (3.16) in the form

$$
1-F(t)=\{A(t)-B(t)\} /\{1-B(t)\}
$$


From equation (3.17), $\{A(t)-B(t)\}$ is positive and monotonic increasing for $0<t \leqq t_{0}$ and also $\{1-B(t)\}$ is positive and monotonic decreasing for $0<t \leqq t_{0}$. Hence $1-F(t)$ is monotonic increasing and from this $F^{\prime}<0$ for $0<t \leqq t_{0}$. It is easy to check that $F^{\prime}(0)=B^{\prime}(0)-A^{\prime}(0)<0$ and so complete the requirement that $F^{\prime}<0$ for $0 \leqq t \leqq t_{0}$.

In particular, we shall want to use this result in cases where

$$
A(t)=\sum_{r=1}^{N} \alpha_{r} t^{r}, \quad B(t)=\sum_{r=1}^{N} \beta_{r} t^{r}
$$

with $\alpha_{r}>\beta_{r} \geqq 0$.

\section{Additional results for cylinder-cylinder comparison}

If we replace $M(t)$ by $M_{1}(\rho, t)$ in equation (2.12), to show the dependence on $\rho$, then it is easy to check that

$$
M_{1}(\rho, t) M_{1}(1 / \rho, \rho t)=\tau^{2}
$$

For $\rho>1$, we have $0<\rho_{1}<1$, where $\rho_{1}=1 / \rho$, so if $M_{1}(\rho, t)$ decreases with $t$ for $t>0$, then $M_{1}\left(\rho_{1}, T\right)$ increases with $T$ for $T=\rho t>0$. From this, if we can show that $M^{\prime}<0$ for $t>0$ and $\rho>1$, then it follows that $M^{\prime}>0$ for $t>0$ and $0<\rho<1$. Thus it is enough to discuss the case where $\rho>1$.

By a similar argument, we can reduce the range of $\rho$ that need be considered to an interval $1<\rho \leqq \rho_{0}$. For a larger value of $\rho$, say $\rho=\rho_{2}$, we can write $\rho_{2}=\rho_{3}^{N}$, where $1<\rho_{3} \leqq \rho_{0}$ and $N$ is an integer greater than 1 . Then

$$
\begin{aligned}
\tau^{N-1} M_{1}\left(\rho_{2}, t\right) & =\left(\tau^{N}\right) \prod_{r=0}^{N-1}\left[G_{1}\left(\rho_{3}^{N-r} t\right) / G_{1}\left(\rho_{3}^{N-r-1} t\right)\right] \\
& =\prod_{r=0}^{N-1}\left[M_{1}\left(\rho_{3}, T_{r}\right)\right]
\end{aligned}
$$

where $T_{r}=\rho_{3}^{N-r-1} t$. If $M_{1}\left(\rho_{3}, t\right)$ is monotonic decreasing for $t>0$, then each factor $M_{1}\left(\rho_{3}, T_{r}\right)$ is monotonic decreasing for $t>0$ and consequently $M_{1}\left(\rho_{2}, t\right)$ is monotonic decreasing. Because of this, we need not worry about the possibility that $\rho t$ is large even although $t$ is small; by taking $\rho_{0}=1.05$, say, we can ensure that $\rho t$ and $t$ are both small when $t$ is small.

From equation (2.12),

$$
\begin{aligned}
M^{\prime}(t) & =\tau\left\{\rho H_{1}(\rho t) G_{1}(t)-H_{1}(t) G_{1}(\rho t)\right\} /\left\{G_{1}(t)\right\}^{2} \\
& =(1 / t) M(t)\{K(\rho t)-K(t)\}
\end{aligned}
$$

where

$$
K(t)=t H_{1}(t) / G_{1}(t)
$$

From equation (4.4), if we can show that $K(t)$ decreases with $t$, then $K(\rho t)<K(t)$ for $\rho>1$ and $M^{\prime}(t)<0$ for $\rho>1$. We have

$$
K^{\prime}(t)=\left[\left\{\left(H_{1}+t H_{1}^{\prime}\right) G_{1}-t H_{1}^{2}\right\} / G_{1}^{2}\right]
$$


and this function is certainly negative if $H_{1}+t H_{1}^{\prime}$ is negative. Using equation (2.6),

$$
H_{1}+t H_{1}^{\prime}=4 \sum_{p=1}^{\infty}\left(1-t \lambda_{p}^{2}\right) \exp \left(-\lambda_{p}^{2} t\right) .
$$

Each of the factors $1-t \lambda_{\mathrm{p}}^{2}$ is negative for $t>t_{0}=1 / \lambda_{1}^{2}$, while for $t=t_{0}$ the first term in the summation is zero and all the others are negative. Hence $H_{1}+t H_{1}^{\prime}<0$ for $t \geqq t_{0}$ and consequently $K^{\prime}(t)<0$ for $t \geqq t_{0}$. (This is the argument used by Clements and Edelstein in obtaining the inequalities (2.14) and (2.15).)

For $t$ small, we can use $K^{*}(t)=t H_{1}^{*}(t) / G_{1}^{*}(t)$ as an approximation to $K(t)$. If we put $u=\sqrt{ } t$ and write

$$
\begin{aligned}
t H_{1}^{*}(t) & =(2 u / \sqrt{ } \pi)\left[1-e_{1} u-e_{2} u^{2}-e_{3} u^{3}-\ldots\right], \\
G_{1}^{*}(t) & =(4 u / \sqrt{ } \pi)\left[1-d_{1} u-d_{2} u^{2}-d_{3} u^{3}-\ldots\right],
\end{aligned}
$$

then the coefficients $d_{r}$ and $e_{r}$ are all positive, with

$$
e_{r}=(r+1) d_{r}=\{\Gamma(1 / 2) / \Gamma[(r+1) / 2]\} a_{r} .
$$

Since $e_{r}>d_{r}>0$ for all $r$, we can use the result at the end of Section 3 to deduce that $K^{*}(t)$ is monotonic decreasing for $t$ small. As $t \rightarrow 0, K^{*}(t) \rightarrow 1 / 2$ from below, a result which agrees with the numerical values in Table 1 .

If we accept that these results for $K^{*}(t)$ give the behaviour of $K(t)$ satisfactorily for $0<t \leqq 0.08$ and that $K^{\prime}(t)<0$ for $t \geqq t_{0}=1 / \lambda_{1}^{2}=0.173$, we still have a gap between $t=0.08$ and $t=0.173$. One way of bridging the gap is to note (from equation (4.6)) that the sign of $K^{\prime}(t)$ is the same as that of $L(t)$, where

$$
L(t)=\left(H_{1}+t H_{1}^{\prime}\right) G_{1}-t H_{1}^{2} \text {. }
$$

We know that for $t>0$

$G_{1}$ is positive and monotonic increasing,

$H_{1}$ is positive and monotonic decreasing,

and since

$$
H_{1}^{\prime}=-4 \sum_{p=1}^{\infty} \lambda_{p}^{2} \exp \left(-\lambda_{p}^{2} t\right)
$$

we can add that $H_{1}^{\prime}$ is negative and monotonic increasing. From these properties, if $0<t_{2} \leqq t \leqq t_{1}$, then

$$
Y\left(t_{2}, t_{1}\right) \leqq L(t) \leqq Y\left(t_{1}, t_{2}\right),
$$

where

$$
Y\left(t_{1}, t_{2}\right)=t_{2} G_{1}\left(t_{2}\right) H_{1}^{\prime}\left(t_{1}\right)+G_{1}\left(t_{1}\right) H_{1}\left(t_{2}\right)-t_{2}\left[H_{1}\left(t_{1}\right)\right]^{2} .
$$

Hence if $Y\left(t_{1}, t_{2}\right)<0$, then $L(t)<0$ for $t_{2} \leqq t \leqq t_{1}$ and consequently $K^{\prime}(t)<0$ over this interval. This provides a straightforward way of extending the range of validity, at the expense of some trial-and-error arithmetic in choosing suitable values for $t$, and $t_{2}$. Table 2 shows values of $G_{1}, H_{1}$ and $H_{1}^{\prime}$ for different values of $t$ and corresponding values of $Y$ for consecutive intervals. They verify that $Y\left(t_{n-1}, t_{n}\right)<0$ for each interval 
Table 2. Values of $G_{1}, H_{1}, H_{1}^{\prime}$ and $Y$

\begin{tabular}{rlllll}
\hline$n$ & \multicolumn{1}{c}{$t_{n}$} & $G_{1}\left(t_{n}\right)$ & $H_{1}\left(t_{n}\right)$ & $H_{1}^{\prime}\left(t_{n}\right)$ & $Y\left(t_{n-1}, t_{n}\right)$ \\
\hline 0 & 0.173 & 0.7449 & 1.4921 & -9.1384 & \\
1 & 0.16 & 0.7248 & 1.6162 & -10.1021 & -0.2122 \\
2 & 0.15 & 0.7081 & 1.7215 & -10.9815 & -0.2144 \\
3 & 0.14 & 0.6904 & 1.8363 & -12.0138 & -0.1759 \\
4 & 0.13 & 0.6714 & 1.9624 & -13.2456 & -0.1321 \\
5 & 0.12 & 0.6511 & 2.1021 & -14.7415 & -0.0857 \\
6 & 0.11 & 0.6293 & 2.2584 & -16.5925 & -0.0361 \\
7 & 0.105 & 0.6175 & 2.3441 & -17.6904 & -0.1363 \\
8 & 0.1 & 0.6058 & 2.4356 & -18.9308 & -0.1172 \\
9 & 0.095 & 0.5934 & 2.5337 & -20.3408 & -0.0958 \\
10 & 0.09 & 0.5805 & 2.6393 & -21.9538 & -0.0742 \\
11 & 0.085 & 0.5670 & 2.7536 & -23.8121 & -0.0518 \\
12 & 0.08 & 0.5529 & 2.8780 & -25.9701 & -0.0281 \\
13 & 0.076 & 0.5412 & 2.9857 & -27.9590 & -0.0468 \\
14 & 0.072 & 0.5290 & 3.1020 & -30.2308 & -0.0280 \\
15 & 0.068 & 0.5164 & 3.2280 & -32.8441 & -0.0081 \\
\hline
\end{tabular}

and consequently $K^{\prime}(t)<0$ for $0.068 \leqq t \leqq t_{0}$, thus bridging the gap mentioned above.

Once it has been established that $K^{\prime}(t)<0$ for $t>0$, it follows that $M^{\prime}(t)<0$ for $\rho>1$ and $M^{\prime}(t)>0$ for $0<\rho<1$. (For $\rho=1, M(t)=\tau$ and $M^{\prime}(t)=0$.)

\section{Diffusion into spherical organism}

For a spherical organism of radius $b$ and diffusion coefficient $q$, we can take the concentration of the toxic material at time $t$ and distance $r$ from the centre as $C_{2}(r, t)$, where

$$
\frac{\partial C_{2}}{\partial t}=q\left(\frac{\partial^{2} C_{2}}{\partial r^{2}}+\frac{2}{r} \frac{\partial C_{2}}{\partial r}\right)
$$

with

$$
C_{2}(r, 0)=0, \quad C_{2}(b, t)=c .
$$

As in Section 2, if we solve by separation of variables and average over $r$, the average concentration is

$$
\tilde{C}_{2}(t)=c G_{2}\left(t_{3}\right)
$$

where $t_{3}=q t / b^{2}$ and

$$
G_{2}\left(t_{3}\right)=1-6 \sum_{p=1}^{\infty}\left(1 / \mu_{p}\right)^{2} \exp \left(-\mu_{p}^{2} t_{3}\right) .
$$

In this case, the eigenvalues are $\mu_{p}=p \pi$ and $G_{2}\left(t_{3}\right)$ is a standardised solution for a sphere with $q=1, b=1$ and $c=1$. As before, $G_{2}$ is a monotonic increasing function of $t_{3}$, with $G_{2}(0)=0$ and $G_{2} \rightarrow 1$ as $t_{3} \rightarrow \infty$. The ratio $t_{3} / t=q / b^{2}$ is again the time scale factor required to relate the given problem to the standardised solution. From equation 
(5.4),

$$
H_{2}\left(t_{3}\right)=G_{2}^{\prime}\left(t_{3}\right)=6 \sum_{p=1}^{\infty} \exp \left(-\mu_{p}^{2} t_{3}\right)
$$

and $H_{2}\left(t_{3}\right)$ is monotonic decreasing, with $H_{2} \rightarrow 0$ as $t_{3} \rightarrow \infty$.

To obtain approximations to $G_{2}$ and $H_{2}$ for $t_{3}$ small, we can use the same procedure as in Section 3. If $h_{2}(s)$ is the Laplace transform of $H_{2}$, then instead of equation (3.2) we get

$$
h_{2}(s)=6 \sum_{p=1}^{\infty}\left(s+\mu_{p}^{2}\right)^{-1}=6 \sum_{p=1}^{\infty}\left(s+p^{2} \pi^{2}\right)^{-1}
$$

Using the summation,

$$
\sum_{p=1}^{\infty} \frac{2 z}{z^{2}+p^{2} \pi^{2}}=\frac{\cosh z}{\sinh z}-\frac{1}{z}
$$

we can write

$$
h_{2}(s)=(3 / \sqrt{ } s) \operatorname{coth} \sqrt{ } s-(3 / s),
$$

for $\operatorname{Re}(s)>0$. For $s$ large and positive, and any positive integer $n$,

$$
\operatorname{coth} \sqrt{ } s=\{1+\exp (-2 \sqrt{ } s)\} /\{1-\exp (-2 \sqrt{ } s)\}=1+o\left(s^{-n}\right),
$$

so for $s$ large we get

$$
h_{2}(s)=(3 / \sqrt{ } s)-(3 / s)+o\left(s^{-n}\right)
$$

Hence we can expect the behaviour of $\mathrm{H}_{2}(t)$, for $t$ small, to be given approximately by

$$
H_{2}^{*}(t)=3 / \sqrt{ }(\pi t)-3
$$

with

$$
G_{2}^{*}(t)=6 \underset{v}{ }(t / \pi)-3 t
$$

as the corresponding approximation to $G_{2}(t)$. Similarly, we can use $K_{2}^{*}(t)=$ $t H_{2}^{*}(t) / G_{2}^{*}(t)$ as an approximation to

$$
K_{2}(t)=t H_{2}(t) / G_{2}(t)
$$

Table 3 shows numerical values for $G_{2}(t), G_{2}^{*}(t), H_{2}(t), H_{2}^{*}(t), K_{2}(t)$ and $K_{2}^{*}(t)$. In this

Table 3. Comparison of $G_{2}(t), H_{2}(t)$ and $K_{2}(t)$ with power series approximations for $t$ small.

\begin{tabular}{ccccccc}
\hline$t$ & $G_{2}(t)$ & $G_{2}^{*}(t)$ & $H_{2}(t)$ & $H_{2}^{*}(t)$ & $K_{2}(t)$ & $K_{2}^{*}(t)$ \\
\hline 0.02 & 0.4187 & 0.4187 & 8.9683 & 8.9683 & 0.4284 & 0.4284 \\
0.04 & 0.5570 & 0.5570 & 5.4628 & 5.4628 & 0.3923 & 0.3923 \\
0.06 & 0.6492 & 0.6492 & 3.9099 & 3.9099 & 0.3614 & 0.3614 \\
0.08 & 0.7175 & 0.7175 & 2.9842 & 2.9841 & 0.3327 & 0.3327 \\
0.10 & 0.7705 & 0.7705 & 2.3529 & 2.3524 & 0.3054 & 0.3053 \\
0.12 & 0.8127 & 0.8126 & 1.8884 & 1.8860 & 0.2788 & 0.2785 \\
\hline
\end{tabular}


case, the power series approximations give 4-figure accuracy up to about $t=0.10$ and are beginning to diverge at $t=0.12$. Comparing Table 3 and Table $1, G_{2}$ increases more rapidly than $G_{1}$, for small values of $t$, while $H_{2}$ and $K_{2}$ fall off more rapidly than $H_{1}$ and $K$.

Clearly, $K_{2}(t)$ is analogous to $K(t)$ and in fact the two functions have very similar properties. Writing

$$
\begin{aligned}
K_{2}^{*}(t) & =\{3 \sqrt{ }(t / \pi)-3 t\} /\{6 \sqrt{ }(t / \pi)-3 t\} \\
& =(1 / 2)-(1 / 4)\{\sqrt{ }(\pi t)\} /\{1-(1 / 2) \sqrt{ }(\pi t)\},
\end{aligned}
$$

it is evident that $K_{2}^{*} \rightarrow(1 / 2)$ as $t \rightarrow 0$ and also that $K_{2}^{*}$ decreases monotonically with $t$ for $0<t \leqq 0.12$. Also, the same argument as for $K(t)$ gives

$$
K_{2}^{\prime}(t)<0 \text { for } t \geqq\left(1 / \mu_{1}\right)^{2}=0.1013 \text {. }
$$

Combining the results in the same way as for $K(t)$ leads to the conclusion that $K_{2}(t)$ is monotonic decreasing for $t>0$. Once this has been established the results for a sphere-sphere comparison, where the parasite and host are both taken as spherical, would go through in the same way as for the cylinder-cylinder comparison, with $\rho=1$ as the critical value for $\rho$.

\section{Cylinder-sphere comparison: preliminary discussion}

Following Clements (2), we take the host organism as cylindrical. For radius $a$ and diffusion coefficient $k$, the average concentration at time $t$ is given by equations (2.3) to (2.5). For the parasite, we can apply the results for a sphere of radius $b$ and diffusion coefficient $q$ in Section 5. If we set

$$
\rho=t_{1} / t_{3}=(k / q)(b / a)^{2},
$$

then $\rho$ is again the ratio of the time scales appropriate to the two organisms and we have

$$
\begin{aligned}
\bar{C}_{2} & =\text { average concentration in parasite }=c G_{2}\left(t_{3}\right), \\
\bar{C} & =\text { average concentration in host }=c G_{1}\left(\rho t_{3}\right) .
\end{aligned}
$$

Hence

$$
\begin{aligned}
M & =\text { value of } \bar{C} \text { when } \bar{C}_{2} \text { reaches value } \tau \\
& =c G_{1}\left(\rho T_{3}\right)=\tau G_{1}\left(\rho T_{3}\right) / G_{2}\left(T_{3}\right),
\end{aligned}
$$

where $c G_{2}\left(T_{3}\right)=\tau$, i.e. $t_{3}=T_{3}$ when $\bar{C}_{2}=\tau$. As before, $T_{3} \rightarrow 0$ as $c \rightarrow \infty$ and $T_{3} \rightarrow \infty$ as $c \rightarrow \tau$ from above, with $T_{3}$ decreasing as $c$ increases. It is again easier to treat $M$ as a function of $T_{3}$ and to work with

$$
M(t)=\tau G_{1}(\rho t) / G_{2}(t) .
$$

With this notation the main results given by Clements are

(i) $M \rightarrow(2 \tau / 3) \sqrt{ } \rho$ as $t \rightarrow 0$,

(ii) $M^{\prime}>0$ for $0<\rho<1$ and $t>T_{0}$,

(iii) $M^{\prime}<0$ for $\rho>\rho^{*}>1$ and $t>T_{0}$, 
where

$$
\rho^{*}=\left(\mu_{1} / \lambda_{1}\right)^{2}=1.707, \quad T_{0}=\left\{\ln \left(\mu_{1}^{2} / \rho \lambda_{1}^{2}\right)\right\} /\left(\mu_{1}^{2}-\rho \lambda_{1}^{2}\right) .
$$

Clements conjectured that $M^{\prime}>0$ for $\rho<1$ and that $M^{\prime}<0$ for $\rho>1$, for all positive values of $t$. The results below indicate that $M^{\prime}>0$ for $0<\rho \leqq \rho^{*}$ and that $M^{\prime}<0$ for $\rho \geqq 4$, for $t>0$. For the intermediate values of $\rho$, i.e. for $\rho^{*}<\rho<4$, it appears that $M^{\prime}>0$ for small values of $t$ but the sign of $M^{\prime}$ changes at some stage and $M^{\prime}$ is negative for sufficiently large values of $t$.

One of our critical values for $\rho$ comes from the behaviour of $M(t)$ when $t$ is small. For small values of $t$ and $\rho t$, we can use the power series approximations to $G_{1}$ and $G_{2}$ to obtain a corresponding approximation to $M$. For convenience, let $u=\sqrt{ } t$ and $\sigma=\sqrt{ } \rho$. Then

$$
\begin{aligned}
M_{2}^{*} & =\tau G_{1}^{*}(\rho t) / G_{2}^{*}(t)=\text { approximation to } M \text { for } t \text { and } \rho t \text { small } \\
& =(2 \sigma \tau / 3)\left(1-d_{1} \sigma u-d_{2} \sigma^{2} u^{2}-\ldots\right) /\{1-(1 / 2) u \sqrt{ } \pi\}
\end{aligned}
$$

where the coefficients $d_{1}, d_{2}, \ldots$ are defined by equations (3.8) and (4.10). More conveniently,

$$
d_{1}=8 d_{3}=(96 / 13) d_{5}=(1 / 4) \sqrt{ } \pi, \quad d_{2}=(8 / 5) d_{4}=(1120 / 1073) d_{6}=1 / 12 .
$$

All the coefficients $d_{r}$ are positive and for $\rho>4$ the result at the end of Section 3 can be used to deduce that $M_{2}^{*}$ decreases with $t$, for $t$ small. More generally, if we expand $M_{2}^{*}$ in powers of $u$ and write

$$
M_{2}^{*}=(2 \sigma \tau / 3)\left(1+B_{1} u+B_{2} u^{2}+\ldots\right)
$$

then

$$
\begin{aligned}
B_{1} & =(1 / 4)(2-\sigma) \sqrt{ } \pi, \quad B_{2}=(\pi / 8)(2-\sigma)-\left(\sigma^{2} / 12\right), \\
B_{n+1} & =(1 / 2) B_{n} \sqrt{ } \pi-\sigma^{n+1} d_{n+1}, \quad(n=2,3, \ldots) .
\end{aligned}
$$

This form for $M_{2}^{*}$ confirms that $M \rightarrow(2 \tau / 3) \sqrt{ } \rho$ as $t \rightarrow 0$ but it also shows that the approach to the limiting value is from below for $\sigma \geqq 2$, i.e. for $\rho \geqq 4$, and from above for $\rho<4$. Thus the conjecture that $M^{\prime}<0$ for $\rho>1$ for all values of $t$ has to be abandoned, since we have $M^{\prime}>0$ for small values of $t$ when $1<\rho<4$. Indeed, this conjecture is not consistent with the limiting values when $\rho$ lies between 1 and $9 / 4$. For these values of $\rho$ the limiting value as $t \rightarrow 0$ is less than $\tau$ and the limiting value as $t \rightarrow \infty$ is equal to $\tau$ so $M$ must increase at some stage as $t$ goes from zero to infinity.

The behaviour of $M_{2}^{*}$ as $t \rightarrow 0$ shows why $\rho=4$ has to be considered as one of the critical values for $\rho$. The other critical value, $\rho=\rho^{*}$, comes in from the behaviour of $M$ as $t \rightarrow \infty$ but some preliminary results are needed before we discuss the critical values in detail. From equation (6.5),

$$
M^{\prime}(t)=\tau N(t) /\left\{G_{2}(t)\right\}^{2}=(1 / t) M(t)\left\{K(\rho t)-K_{2}(t)\right\}
$$

where

$$
N(t)=\rho G_{2}(t) H_{1}(\rho t)-H_{2}(t) G_{1}(\rho t)
$$

and $K(t)$ and $K_{2}(t)$ are the functions already defined in equations (4.5) and (5.12). Thus for $t>0$

$$
\operatorname{sgn} M^{\prime}(t)=\operatorname{sgn} N(t)=\operatorname{sgn}\left\{K(\rho t)-K_{2}(t)\right\}
$$


From Section $4, K^{\prime}(t)<0$ for $t>0$ and hence if $K\left(\rho_{0} t\right)<K_{2}(t)$ for $t>0$ for a specified value $\rho=\rho_{0}$ then we can assert that for $\rho>\rho_{0}$

$$
K(\rho t)<K\left(\rho_{0} t\right)<K_{2}(t) \text { for } t>0 .
$$

This means that if $M^{\prime}(t)<0$ for $t>0$ and $\rho=\rho_{0}$, then $M^{\prime}(t)<0$ for $t>0$ and $\rho>\rho_{0}$. In particular, we want to use this argument with $\rho_{0}=4$.

A similar argument shows that if $M^{\prime}(t)>0$ for $t>0$ and $\rho=\rho_{0}$, then $M^{\prime}(t)>0$ for $t>0$ and $0<\rho<\rho_{0}$. We want to use this idea with $\rho_{0}=\rho^{*}$. Note that for $\rho=\rho^{*}$ and $\rho=4$ we can take $t$ and $\rho t$ as being of the same order of magnitude and, for example, we can use equations (6.7) and (6.9) when $u$ is small.

\section{Cylinder-sphere comparison for $\rho>\rho^{*}$}

In considering the behaviour of $H_{1}$ and $H_{2}$ for large values of $t$ it is worth noting that to two decimal places

$$
\begin{array}{lll}
\lambda_{1}^{2}=5.78, & \lambda_{2}^{2}=30.47, & \lambda_{3}^{2}=74.89, \ldots \\
\mu_{1}^{2}=9.87, & \mu_{2}^{2}=39.48, & \mu_{3}^{2}=88.83, \ldots
\end{array}
$$

so that even for $t=1$ the later terms in the series are small compared with $\exp \left(-\lambda_{1}^{2} t\right)$ and $\exp \left(-\mu_{1}^{2} t\right)$. This implies that for $\rho>1$ and $t \geqq 1$ the dominant terms in $H_{1}(\rho t)$ and $H_{2}(t)$ are $4 \exp \left(-\rho \lambda_{1}^{2} t\right)$ and $6 \exp \left(-\mu_{1}^{2} t\right)$, respectively. Since $4 \exp \left(-\lambda_{1}^{2}\right)=0.0123$ and $6 \exp \left(-\mu_{1}^{2}\right)=3.1 \times 10^{-4}$, the dominant terms are small compared with unity and we can take $G_{1}(\rho t)$ and $G_{2}(t)$ as approximately 1 in considering the expressions which occur in $N(t)$. Thus we can take $U=4 \rho \exp \left(-\rho \lambda_{1}^{2} t\right)$ as the dominant term in $\rho G_{2}(t) H_{1}(\rho t)$, with $V=6 \exp \left(-\mu_{1}^{2} t\right)$ as the dominant term in $H_{2}(t) G_{1}(\rho t)$. If $\rho \lambda_{1}^{2}>\mu_{1}^{2}$, then as $t$ increases $U-V$ will eventually become negative and we can expect $N(t)$ and $M^{\prime}(t)$ to become negative. This means that for $\rho>\rho^{*}$ we should have $M^{\prime}<0$ for $t$ sufficiently large. On the other hand, if $\rho \lambda_{1}^{2}<\mu_{1}^{2}, U-V$ will be positive eventually and we should have $M^{\prime}>0$ for $t$ sufficiently large.

For $\rho>\rho^{*}$, we can make this argument more precise by comparing $\rho H_{1}(\rho t)$ with $H_{2}(t)$ and $G_{1}(\rho t)$ with $G_{2}(t)$. If we can show that for $t \geqq T^{*}$

$$
0<G_{2}(t)<G_{1}(\rho t)<1 \text { and } 0<\rho H_{1}(\rho t)<\dot{H}_{2}(t) \text {, }
$$

then it follows that $N(t)<0$ for $t \geqq T^{*}$ and hence $M^{\prime}(t)<0$ for $t \geqq T^{*}$. A useful preliminary result is that $\mu_{1} / \lambda_{1}>\mu_{n} / \lambda_{n}$ for $n=2,3, \ldots$ This follows from equation (3.3) and the properties of $\varepsilon(n)$ mentioned at that point, since

$$
\begin{aligned}
\mu_{1} \lambda_{n}-\lambda_{1} \mu_{n}= & \pi^{2}[\{n-(1 / 4)+\varepsilon(n)\}-n\{1-(1 / 4)+\varepsilon(1)\}] \\
= & \pi^{2}[n\{(1 / 4)-\varepsilon(1)\}+\varepsilon(n)-(1 / 4)] \\
& >\pi^{2}[(0.23) n-0.25]>0 .
\end{aligned}
$$

Indeed, it can be checked that $\left\{\mu_{n} / \lambda_{n}\right\}$ is a monotonic decreasing sequence, with limit 1 as $n \rightarrow \infty$, although this result is not required in the discussion below.

If we write $\nu_{n}=\mu_{n} / \lambda_{n}$ and $Z_{n}=\rho \lambda_{n}^{2}-\mu_{n}^{2}$, then we can use the inequality above to deduce that, for $n=2,3, \ldots, Z_{n}>Z_{1}>0$ for $\rho>\rho^{*}$. Note that

$$
\begin{aligned}
Z_{n}-Z_{1}= & \lambda_{n}^{2}\left(\rho-\nu_{n}^{2}\right)-\lambda_{1}^{2}\left(\rho-\nu_{1}^{2}\right) \\
& >\lambda_{n}^{2}\left(\rho-\nu_{1}^{2}\right)-\lambda_{1}^{2}\left(\rho-\nu_{1}^{2}\right)>0,
\end{aligned}
$$


since $\nu_{n}^{2}<\nu_{1}^{2}=\rho^{*}$ and $\lambda_{n}>\lambda_{1}>0$. If we put

$$
X_{p}=\left(6 / \mu_{p}^{2}\right) \exp \left(-\mu_{p}^{2} t\right), \quad Y_{p}=\left(4 / \lambda_{p}^{2}\right) \exp \left(-\rho \lambda_{p}^{2} t\right)
$$

then

$$
X_{p} / Y_{p}=\left(3 / 2 \nu_{p}^{2}\right) \exp \left\{Z_{p} t\right\}
$$

This ratio increases with $t$ and for $p=2,3, \ldots$

$$
X_{p} / Y_{p}>\left(3 / 2 \nu_{1}^{2}\right) \exp \left\{Z_{1} t\right\}=X_{1} / Y_{1}
$$

From equation (7.4), with $p=1, X_{1}=Y_{1}$ for $t=\tau_{1}$, where

$$
\tau_{1}=\left(1 / Z_{1}\right) \ln (2 \rho * / 3)
$$

and $X_{1}>Y_{1}$ for $t>\tau_{1}$. From equation (7.5), it follows that $X_{p}>Y_{p}$ for $t \geqq \tau_{1}$, for $p=2,3, \ldots$, and hence

$$
1-G_{2}(t)=\sum_{p=1}^{\infty} X_{p}>\sum_{p=1}^{\infty} Y_{p}=1-G_{1}(\rho t)
$$

Thus, for $t \geqq \tau_{1}$,

$$
0<G_{2}(t)<G_{1}(\rho t)<1 .
$$

In similar fashion, we can put

$$
Q_{p}=6 \exp \left(-\mu_{p}^{2} t\right), \quad R_{p}=4 \rho \exp \left(-\rho \lambda_{p}^{2} t\right)
$$

and write

$$
Q_{p} / R_{p}=(3 / 2 \rho) \exp \left(Z_{p} t\right)
$$

As before, this ratio increases with $t$ and for $p=2,3, \ldots$.

$$
Q_{p} / R_{p}>Q_{1} / R_{1} \geqq 1 \text { for } t \geqq \tau_{2} \text {, }
$$

where

$$
\tau_{2}=\left(1 / Z_{1}\right) \ln (2 \rho / 3)
$$

Hence for $t \geqq \tau_{2}$

$$
H_{2}(t)=\sum_{p=1}^{\infty} Q_{p}>\sum_{p=1}^{\infty} R_{p}=\rho H_{1}(\rho t)>0 \text {. }
$$

Since $\rho>\rho^{*}, \tau_{2}>\tau_{1}$ and the inequalities (7.7) and (7.12) are both valid for $t \geqq \tau_{2}$. It follows that $N(t)<0$ for $t \geqq \tau_{2}$ and consequently

$$
M^{\prime}(t)<0, \text { for } \rho>\rho^{*} \text { and } t \geqq \tau_{2} \text {. }
$$

This result is similar to one given by Clements and cited as result (iii) in Section 6 . The only difference is that $T_{0}$ and $\tau_{2}$ are not the same. From equation (6.6), we can re-write $T_{0}$ as

$$
T_{0}=\left(1 / Z_{1}\right) \ln \left(\rho / \rho^{*}\right)
$$

and compare it with $\tau_{2}$. The two expressions have a similar structure, with $\rho^{*}$ in $T_{0}$ 
replaced by $3 / 2$ in $\tau_{2}$. Since $\rho^{*}>3 / 2$, we have $0<T_{0}<\tau_{2}$, which makes Clements' result valid for a wider range of $t$. The difference becomes most conspicuous as $\rho \rightarrow \rho^{*}$ from above, for in this limiting case $T_{0} \rightarrow(1 / \pi)^{2}$ whereas $\tau_{2} \rightarrow \infty$. It will be shown later than $N(t)>0$ for $t>0$ when $\rho=\rho^{*}$, a result which is difficult to reconcile with the finite limit for $T_{0}$ as $\rho \rightarrow \rho^{*}$.

For $\rho=4$, we get $\tau_{2}=\{\ln (8 / 3)\} /\left(4 \lambda_{1}^{2}-\mu_{1}^{2}\right)=0.074$ and thus have $M^{\prime}(t)<0$ for $t \geqq$ 0.074 . For small values of $t$, we can use equation (6.7) with $\sigma=2$. In practice, the approximation used was

$$
M_{2}^{*}=(4 \tau / 3)-(4 \tau / 3)\left\{\sum_{=2}^{6}(2 u)^{r} d_{r}\right\} /\{1-(1 / 2) u \sqrt{ } \pi\}
$$

which is clearly a monotonic decreasing function of $u$ (and hence of $t$ ) for $u>0$. This approximation was compared with exact values of $M(t)$ for $0.01 \leqq t \leqq 0.06$ and at the lower end of the range the results agreed to five decimal places. For $t=0.05,0.055$ and 0.06 , the corresponding values were $1.289,1.282$ and 1.274 for $(1 / \tau) M$ and 1.290 , 1.284 and 1.277 for $(1 / \tau) M_{2}^{*}$.

If we take the result for small $t$ as valid up to $t=0.05$, there is again a gap which can be bridged by the use of inequalities, as in Section 4 . Since $K$ and $K_{2}$ are both monotonic decreasing functions of $t$, if we consider an interval $0<t_{1} \leqq t \leqq t_{2}$ and let

$$
X\left(t_{1}, t_{2}\right)=K\left(\rho t_{1}\right)-K_{2}\left(t_{2}\right)
$$

then over this interval

$$
X\left(t_{2}, t_{1}\right) \leqq K(\rho t)-K_{2}(t) \leqq X\left(t_{1}, t_{2}\right) .
$$

Thus if $X\left(t_{1}, t_{2}\right)<0$, then $K(\rho t)-K_{2}(t)<0$ for $t_{1} \leqq t \leqq t_{2}$ and this implies that $M^{\prime}<0$ throughout the interval (using equation (6.13)). For $\rho=4$, this technique was used in turn for the intervals $[0.04,0.0 .045],[0.045,0.05],[0.05,0.06]$, and $[0.06,0.08]$ and the corresponding values for $X\left(t_{1}, t_{2}\right)$ were $-0.0275,-0.0358,-0.0373$, and -0.0424 . Thus the bridging technique gives $M^{\prime}<0$ for $0.04 \leqq \mathrm{t} \leqq 0.08$.

Combining this with the earlier results, we get $M^{\prime}<0$ for $t>0$ when $\rho=4$ and the argument near the end of Section 6 allows this result to be extended to $\rho>4$.

\section{Cylinder-sphere comparison for $\rho=\rho^{*}$}

For $\rho=\rho^{*}$, there is a break-down in the argument used in Section 7 when comparing $\rho H_{1}(\rho t)$ with $H_{2}(t)$, since $Z_{1}=0$ for $\rho=\rho^{*}$ and hence $Q_{1} / R_{1}=3 /\left(2 \rho^{*}\right)<1$ for all $t$. For $n \geqq 2, Z_{n}$ is still positive and it can be shown that $Q_{n} / R_{n}>1$ for $t>\tau_{3}$, where $\tau_{3}=\left(1 / Z_{2}\right) \ln \left(2 \rho^{*} / 3\right)=0.0103$. For $t=\tau_{3}, Q_{2}=R_{2}$ and $Q_{n}>R_{n}$ for $n>2$. (The details follow in the same way as in Section 7.) Thus although equation (7.12) is no longer valid, we still have

$$
H_{2}(t)-Q_{1}=\sum_{p=2}^{\infty} Q_{p}>\sum_{p=2}^{\infty} R_{p}=\rho^{*} H_{1}\left(\rho^{*} t\right)-R_{1}
$$

for $t \geqq \tau_{3}$. However, for large values of $t$ the first term in $H_{1}$ and $H_{2}$ is dominant and we 
can expect to have

$$
N(t) \fallingdotseq\left(4 \rho^{*}-6\right) \exp \left(-\mu_{1}^{2} t\right)>0
$$

i.e. $M^{\prime}>0$ for $t$ large.

To put this on a more rigorous basis, we need bounds on the contribution made by the later terms to $H_{1}\left(\rho^{*} t\right), H_{2}(t), G_{1}\left(\rho^{*} t\right)$ and $G_{2}(t)$. If we start with $H_{2}(t)$, a typical term is

$$
Q_{p}=6 \exp \left(-\mu_{p}^{2} t\right)=6 \exp \left(-p^{2} \pi^{2} t\right)=6 E^{p^{2}},
$$

where $E=\exp \left(-\pi^{2} t\right)$. Since we are thinking of what happens when $t$ is large, we can put a lower bound on $t$, say $t \geqq 0.1$, and we then have $0<E \leqq 0.373<1$. We can write

$$
H_{2}=6 E\left(1+E^{3}+E^{8}+\ldots\right)=6 E\left(1+q_{1}\right),
$$

where

$$
q_{1}=E^{3}\left\{1+\sum_{p=3}^{\infty}(E)^{p^{2}-4}\right\}
$$

Now for $p \geqq 3$

$$
p^{2}-4=(p-2)^{2}+4(p-2)>4(p-2)
$$

and hence

$$
q_{1}<E^{3}\left\{1+E^{4}+E^{8}+\ldots\right\}=E^{3} /\left(1-E^{4}\right) .
$$

If we write $F=E^{3} /\left(1-E^{4}\right)$, then $F$ decreases as $t$ increases and $F \leqq 0.0528$ for $t \geqq 0$.1. Thus we can say that $0<q_{1}<v_{1}=0.055$ for $t \geqq 0.1$.

For $H_{1}\left(\rho^{*} t\right)$ it is possible to make use of an inequality similar to (8.4) or alternatively we can use equation (8.1) and say that for $t \geqq \tau_{3}$

$$
0<\rho^{*} H_{1}\left(\rho^{*} t\right)-4 \rho^{*} E<\sum_{p=2}^{\infty} Q_{p}=6 E q_{1}<6 E F
$$

Hence

$$
\rho^{*} H_{1}\left(\rho^{*} t\right)=4 \rho^{*} E\left(1+q_{2}\right),
$$

with $0<q_{2}<\left(6 / 4 \rho^{*}\right) F$. For $t \geqq 0.1$, this gives $0<q_{2}<v_{2}=0.050$.

In the same way, we have

$$
1-G_{2}(t)-\left(6 E / \mu_{1}^{2}\right)=\sum_{p=2}^{\infty}\left\{Q_{p} / \mu_{p}^{2}\right\}<\left(1 / \mu_{2}^{2}\right) \sum_{p=2}^{\infty} Q_{p}
$$

and hence

$$
1-G_{2}(t)=\left(6 E / \mu_{1}^{2}\right)\left(1+q_{3}\right)
$$

with

$$
0<q_{3}<\left(\mu_{1} / \mu_{2}\right)^{2} q_{1}=(1 / 4) q_{1} .
$$

For $t \geqq 0.1$, this gives $0<q_{3}<v_{3}=0.015$. 
Finally we can write

$$
1-G_{1}\left(\rho^{*} t\right)=\left(4 E / \lambda_{1}^{2}\right)\left(1+q_{4}\right)
$$

where

$$
4 E q_{4} / \lambda_{1}^{2}=\sum_{p=2}^{\infty}\left(1 / \rho^{*}\right)\left(R_{p} / \lambda_{p}^{2}\right)<\left(1 / p^{*} \lambda_{2}^{2}\right) \sum_{p=2}^{\infty} R_{p}=4 E q_{2} / \lambda_{2}^{2}
$$

Hence $0<q_{4}<\left(\lambda_{1} / \lambda_{2}\right)^{2} q_{2}=0.1898 q_{2}$, and for $t \geqq 0.1$ we can write $0<q_{4}<v_{4}=0.01$.

Putting these results into equation (6.12),

$$
\begin{aligned}
N(t) & =4 \rho^{*} E\left(1+q_{2}\right)\left\{1-\left(6 E / \mu_{1}^{2}\right)\left(1+q_{3}\right)\right\}-6 E\left(1+q_{1}\right)\left\{1-\left(4 E / \lambda_{1}^{2}\right)\left(1+q_{4}\right)\right\} \\
& =a E+b E^{2}
\end{aligned}
$$

where

$$
\begin{aligned}
& a=4 \rho^{*}\left(1+q_{2}\right)-6\left(1+q_{1}\right) \\
& b=\left(24 / \lambda_{1}^{2}\right)\left(q_{1}+q_{4}+q_{1} q_{4}-q_{2}-q_{3}-q_{2} q_{3}\right) .
\end{aligned}
$$

For $t \geqq 0.1$,

$$
\begin{aligned}
a & >4 \rho^{*}-6\left(1+v_{1}\right)=0.4964 \\
b & >\left(24 / \lambda_{1}^{2}\right)\left(-v_{2}-v_{3}-v_{2} v_{3}\right)=-0.2729, \\
N & >0.4964 E-0.2729 E^{2}>0
\end{aligned}
$$

since $0<E \leqq 0.373$. Thus we conclude that $M^{\prime}>0$ for $\rho=\rho^{*}$ and $t \geqq 0.1$.

For small values of $t$, we can approximate to $M$ by the series $M_{2}^{*}$, as given by equations (6.9) and (6.10). Inserting $\rho=\rho^{*}$ and $\sigma=\sigma^{*}=\sqrt{ } \rho^{*}$, the approximation used was $M_{2}^{*}=(0.8709 \tau) P(u)$, with $u=\sqrt{ } t$ and

$$
P(u)=1+0.3074 u+0.1302 u^{2}-0.0081 u^{3}-0.1589 u^{4}-0.3691 u^{5} .
$$

Numerically, the approximation gives agreement with the exact values of $M$ to about $0.0005 \tau$ at $t=0.07$ and to $0.0003 \tau$ at $t=0.06$. Although the polynomial $P(u)$ has negative coefficients for the $u^{3}, u^{4}$ and $u^{5}$ terms, it is easy to show that $P^{\prime}(u)$ is positive and indeed monotonic increasing for $0 \leqq u \leqq 0.25$, corresponding to $0 \leqq t \leqq 0.0625$. We can take this as evidence that $M^{\prime}>0$ for small values of $t$, say for $0<t \leqq 0.05$.

Equations (7.16) and (7.17) were used to bridge the gap between $t=0.05$ and $t=0.1$. In this case, if we can show that $0<X\left(t_{2}, t_{1}\right)$, where $0<t_{1} \leqq t \leqq t_{2}$ and $X\left(t_{2}, t_{1}\right)=$ $K\left(\rho * t_{2}\right)-K_{2}\left(t_{1}\right)$, then we can deduce that $M^{\prime}>0$ for $t_{1} \leqq t \leqq t_{2}$. In practice, it was more convenient to use $X^{*}\left(t_{3}, t_{1}\right)=K\left(t_{3}\right)-K_{2}\left(t_{1}\right)$ as a criterion, with $t_{3}>\rho^{*} t_{2}$. Because $K$ is monotonic decreasing, $K\left(t_{3}\right)<K\left(\rho^{*} t_{2}\right)$ and hence $X^{*}\left(t_{3}, t_{1}\right)<X\left(t_{2}, t_{1}\right)$, so $K\left(t_{3}\right)>K_{2}\left(t_{1}\right)$ is a sufficient condition to ensure that $M^{\prime}>0$ for $t_{1} \leqq t \leqq t_{2}$. Table 4 shows the numerical values used for $t_{1}, t_{2}$ and $t_{3}$ and the corresponding values of $K_{2}\left(t_{1}\right)$ and $K\left(t_{3}\right)$. Combining these results gives $M>0$ for $0.03 \leqq t \leqq 0.12$, which bridges the gap successfully.

This completes the discussion of the case $\rho=\rho^{*}$. Since $M^{\prime}>0$ for $t>0$ when $\rho=\rho^{*}$, it follows that $M>0$ for $t>0$ when $0<\rho<\rho^{*}$ also. 
Table 4. Values of $t_{1}, t_{2}, t_{3}, K_{2}\left(t_{1}\right)$ and $K\left(t_{3}\right)$.

\begin{tabular}{cccccc}
\hline$t_{1}$ & $t_{2}$ & $\rho^{*} t_{2}$ & $t_{3}$ & $K_{2}\left(t_{1}\right)$ & $K\left(t_{3}\right)$ \\
\hline 0.03 & 0.04 & 0.0683 & 0.07 & 0.4093 & 0.4236 \\
0.04 & 0.05 & 0.0853 & 0.09 & 0.3923 & 0.4092 \\
0.05 & 0.06 & 0.1024 & 0.11 & 0.3764 & 0.3948 \\
0.06 & 0.07 & 0.1195 & 0.12 & 0.3614 & 0.3874 \\
0.07 & 0.08 & 0.1365 & 0.14 & 0.3469 & 0.3724 \\
0.08 & 0.09 & 0.1536 & 0.16 & 0.3328 & 0.3568 \\
0.09 & 0.10 & 0.1707 & 0.18 & 0.3189 & 0.3406 \\
0.10 & 0.12 & 0.2048 & 0.21 & 0.3054 & 0.3156 \\
\hline
\end{tabular}

\section{Sphere-cylinder comparison}

If the host organism is taken as spherical and the parasite as cylindrical, then corresponding results are readily obtainable. We can take the host organism as a sphere of radius $b$ and diffusion coefficient $q$, as before. Then from Section 5,

$$
\bar{C}_{2}(t)=\text { average concentration in host at time } t=c G_{2}\left(t_{3}\right) \text {, }
$$

with $t_{3}=q t / b^{2}$ and $G_{2}$ defined as in equation (5.4). Similarly, for a cylinder of radius $a$ and diffusion coefficient $k$, the average concentration in the parasite at time $t$ is given by equations (2.3), (2.4) and (2.5). If the process terminates when $\bar{C}$ reaches a specified value $\tau_{4}$ and if this occurs when $t_{1}=T_{4}$, then $\tau_{4}=c G_{1}\left(T_{4}\right)$ and the average concentration in the host is $M_{3}$, say, where

$$
M_{3}=c G_{2}\left(\rho_{1} T_{4}\right)=\tau_{4} G_{2}\left(\rho_{1} T_{4}\right) / G_{1}\left(T_{4}\right)
$$

with

$$
\rho_{1}=\left(q a^{2}\right) /\left(k b^{2}\right)=1 / \rho
$$

If we replace $T_{4}$ by $\rho v$ and write $M_{3}$ as a function of $v$, then

$$
M_{3}(v)=\tau_{4} G_{2}(v) / G_{1}(\rho v)=\tau_{4} \tau / M(v),
$$

where $M(v)$ is defined by equation (6.5). Since $M_{3}(v) M(v)=\tau \tau_{4}=$ constant, it follows that

$$
\operatorname{sgn} M_{3}^{\prime}=-\operatorname{sgn} M^{\prime} \text {. }
$$

Note that $T_{4}$ and $v$ increase together and that $T_{4} \rightarrow 0$ as $v \rightarrow 0$. Using the properties of $M$, we can say that

(i) as $c \rightarrow \infty$ and $T_{4} \rightarrow 0, M_{3}$ tends to a limit $l$, where

$$
l=\tau_{4} \tau /\{(2 / 3) \tau \sqrt{ } \rho\}=(3 / 2) \tau_{4} \sqrt{ } \rho_{1}
$$

(ii) $M_{3}^{\prime}>0$ for $v>0$ (or $T_{4}>0$ ) when $\rho \geqq 4$, i.e. when

$$
0<\rho_{1} \leqq 1 / 4
$$

(iii) $M_{3}^{\prime}<0$ for $v>0$ when $0<\rho \leqq \rho^{*}$, i.e. when

$$
\rho_{1} \geqq\left(1 / \rho^{*}\right)=\left(\lambda_{1} / \mu_{1}\right)^{2} \text {. }
$$


Acknowledgement. This work was completed while on study leave in the Faculty of Mathematical Studies, University of Southampton, and I should like to thank the University of Southampton for the facilities put at my disposal during my visit. I should also like to thank Dr. J. C. Clements for sending me a copy of the paper by Clements and Edelstein (2) which initiated the work on this problem.

\section{REFERENCES}

(1) J. Clements, A simple problem in comparative diffusion, Applicable Analysis 6 (1977), 241-251.

(2) J. C. Clements and M. Edelstein, A model for a simple diffusion process, Proc. Nova Scotia Inst. of Sc. 27 (1974-76), 134-143.

(3) A. Erdelyi, W. Magnus, F. Oberhettrnger and F. G. Tricomi, Higher Transcendental Functions, Vol. 2 (McGraw-Hill, New York, 1953).

(4) A. S. B. Holland, Introduction to the Theory of Entire Functions (Academic Press, New York, 1973).

(5) G. N. WATson, A Treatise on the Theory of Bessel Functions, 2nd Edition (Cambridge University Press, London, 1958).

Faculty of Mathematical Studies

UNIVERSITY OF SOUTHAMPTON

AND

Department of Applied Mathematics

FACULTY OF SCIENCE

Australian National University 